\title{
Adaptação dos Inventários de Sexismo Moderno para Portugal: O Inventário de Sexismo Ambivalente e o Inventário de Ambivalência em Relação aos Homens
}

\author{
Adaptation of the Modern Sexism Inventories to Portugal: The Ambivalent \\ Sexism Inventory and the Ambivalence toward Men Inventory
}

\author{
Pedro Alexandre Costa*, Raquel Oliveira, Henrique Pereira \& Isabel Leal \\ Unidade de Investigação em Psicologia e Saúde, ISPA - Instituto Universitário, Lisboa, Portugal
}

\begin{abstract}
Resumo
A relação entre homens e mulheres é única e composta por desigualdade e preconceito dirigido aos membros do sexo oposto. Enquanto a forma hostil de sexismo foi já muito estudada, o sexismo moderno é caracterizado pela simultaneidade de formas hostis e benevolentes de preconceito, e por isso conceitualizado como Sexismo Ambivalente. O objetivo deste estudo foi adaptar o Inventário de Sexismo Ambivalente (ASI) e o Inventário de Ambivalência em relação aos Homens (AMI) para a população Portuguesa, e avaliar as suas propriedades psicométricas. Ambos os inventários foram administrados a 258 estudantes universitários (31\% homens e $69 \%$ mulheres), com uma média de idade de 27 anos. As análises fatoriais confirmatórias revelaram evidência da multidimensionalidade de ambos os inventários, validade fatorial, convergente e discriminante, e fiabilidade interna. Os homens revelaram níveis mais elevados de hostilidade e de benevolência dirigido às mulheres, as mulheres revelaram maiores níveis de hostilidade dirigida aos homens. A hostilidade em relação aos homens aumentou com a idade, enquanto a benevolência diminuiu. O preconceito hostil e benevolente foi maior em pessoas com menos anos de escolaridade. Religiosidade correlacionou significativamente com os índices de sexismo benevolente. Foi assim demonstrada a validade e confiabilidade da adaptação dos Inventários de Sexismo Moderno em Portugal.

Palavras-chave: Sexismo ambivalente, sexismo benevolente, sexismo hostil, análise fatorial confirmatória.
\end{abstract}

\begin{abstract}
The relationship between men and women is rather unique and composed by inequality and prejudice towards members of the opposite sex. Whilst hostile sexism has been well studied, modern sexism is characterized by the simultaneity of hostile and benevolent forms of prejudice, therefore conceptualized as Ambivalent Sexism. The purpose of this study was to adapt the Ambivalent Sexism Inventory (ASI) and the Ambivalence toward Men Inventory (AMI) to the Portuguese population and evaluate their psychometric properties. Both inventories were administrated to 258 university students $(31 \%$ male and $69 \%$ female) with a mean age of 27 years. Confirmatory factor analyses supported the multidimensionality of both inventories and provided evidence of factorial, convergent, and discriminant validity, and internal reliability. Men revealed higher levels of hostility and benevolence toward women, whereas women revealed higher levels of hostility toward men. Hostility toward men increased with age whereas benevolence decreased. Hostile and benevolent prejudice was higher in participants with less education. Religiosity was correlated with benevolent sexism. This study showed the validity and reliability of Modern Sexism Inventories in Portugal.

Keywords: Ambivalent sexism, benevolent sexism, hostile sexism, confirmatory factor analysis.
\end{abstract}

A relação entre homens e mulheres é única, sendo que nenhum outro grupo social tem uma relação de tal forma marcada pela associação entre desigualdade e intimidade (física e psicológica). Esta desigualdade é caracterizada por

\footnotetext{
* Endereço para correspondência: ISPA - Instituto Universitário, Unidade de Investigação em Psicologia e Saúde, Rua Jardim do Tabaco, $\mathrm{n}^{\circ} 34$, Lisboa, Portugal 1149-041. E-mail: pcosta@ispa.pt, hpereira@ubi.pt e ileal@ispa.pt
}

uma relação entre os sexos em que os homens possuem um maior estatuto social do que as mulheres, e um conjunto de crenças e atitudes que diminuem o papel social da mulher (Glick \& Fiske, 1999). Sexismo é então definido como uma forma de preconceito que se baseia em uma atitude negativa inflexível contra as mulheres (Allport, 1954). Este tipo de preconceito não é, contudo, simples ou unidimensional.

Sexismo inclui, por um lado, a forma clássica de hostilidade dirigida às mulheres, cujo objetivo é o de justificar o 
poder masculino sobre as mulheres, e de reforçar os papéis tradicionais de género. Por outro lado, mais recentemente as pesquisas têm evidenciado uma forma de sexismo mais subtil, caracterizada por uma proteção paternalista e aparentemente positiva, com base em justificações de dominância masculina e de papéis de género mais gentis, ainda que reconhecendo a dependência dos homens em relação às mulheres, intitulada sexismo benevolente. Estas duas formas de preconceito sexista servem como mecanismos de manutenção da desigualdade de género nos diversos contextos (Glick \& Fiske, 1997, 2001). A existência simultânea de poder estrutural masculino e de poder diádico feminino leva a ideologias sexistas ambivalentes, compostas por sexismo hostil e benevolente. Os tipos de sexismo hostil e benevolente podem diferir, mas partilham uma mesma condição: ambos se baseiam em papéis de género tradicionais, e servem o propósito de justificar a estrutura social patriarcal (Glick \& Fiske, 1996).

Com a finalidade de medir estes construtos, Glick e Fiske (1996) propuserem o Inventário de Sexismo Ambivalente (Ambivalent Sexism Inventory [ASI]), que avalia as atitudes negativas em relação à mulher, em relação ao poder (paternalismo protetor e dominante), diferenciação de género (complementaridade e competitividade), e heterossexualidade íntima (intimidade heterossexual e hostilidade sexual), permitindo assim determinar o tipo de sexismo hostil ou benevolente.

Em contraste, pesquisadores e teóricos da natureza social do preconceito argumentam que os grupos frequentemente vitimizados e negativamente avaliados desenvolvem estratégias ativas contra a desigualdade de poder exercida sobre eles pelo grupo dominante (Allport, 1954; Tajfel, 1981). Glick e Fiske (1999) mencionam também que este fenómeno foi já observado e caracterizado, uma vez que os indivíduos tendem a lidar com identidades sociais negativas através da competição com outros grupos, ou mantendo atitudes preconceituosas em relação a outros grupos, nomeadamente os grupos dominantes. Desta forma, observando a forma como as mulheres respondem aos fatores sociais associados com os comportamentos de género ambivalentes dos homens, estes autores documentaram a existência de um fenómeno semelhante entre as mulheres, caracterizado por atitudes ambivalentes em relação aos homens, e propuseram uma nova medida, o Inventário de Ambivalência em relação aos Homens (Ambivalence toward Men Inventory [AMI]; Glick \& Fiske, 1999).

As diferenças de poder entre os sexos são racionalizadas através de ideologias paternalistas. Por um lado, o paternalismo dominador é a crença hostil que as mulheres deveriam ser controladas pelos homens. Por outro lado, o paternalismo protetor está associado a uma perspetiva benevolente, na qual os homens são percebidos como protetores das mulheres devido à sua maior autoridade, poder e força física. Este paternalismo protetor é tanto mais evidente quanto maior for a dependência diádica dos homens, por exemplo, da sua esposa, mãe, ou filha
(Glick \& Fiske, 1997). Em contraste, os membros do grupo subordinado tendem a ressentir, de uma forma hostil, o estatuto superior do grupo dominante, o ressentimento do paternalismo (Glick \& Fiske, 1999). Este ressentimento é igualmente evidenciado pela criação de estereótipos negativos em relação ao grupo dominante, nomeadamente pela caracterização dos seus membros como inferiores (por exemplo, as mulheres caracterizarem os homens como bebés quando estes estão doentes), ou pela caracterização destes como tendo características negativas associadas aos seu poder e dominância (por exemplo, os homens serem considerados arrogantes; Glick \& Fiske, 1999). De uma forma benevolente, à semelhança do paternalismo protetor, pode revelar-se uma atitude maternalista, envolvendo a crença de uma fraqueza dos membros do sexo oposto, e que leva a uma atitude protetora das mulheres para com os homens (Glick \& Fiske, 1999). Mais, os estereótipos em relação aos homens e às mulheres constituem também ideologias sobre a diferenciação de género. A diferenciação de género competitiva (e hostil) é reforçada através das crenças negativas em relação ao outro sexo, permitindo que um grupo ganhe autoconfiança com base na afirmação de que são melhores que os membros do sexo oposto. Desta crença decorre uma heterossexualidade obrigatória que é ambivalente como fruto de uma dependência diádica dos homens em relação às mulheres (Glick \& Fiske, 1999).

A estrutura multidimensional destes inventários retrata formas de sexismo moderno que não se concentra somente em aspetos hostis de sexismo, mas também em representam aspetos mais subtis de sexismo que se justificam em crenças aparentemente igualitárias (Glick \& Fiske, 2001). Os estudos de validação de Glick e Fiske $(1996,1999)$ incluíram mais de 2000 participantes em seis estudos com o Inventário de Sexismo Ambivalente, e perto de 1000 participantes em três estudos com o Inventário de Ambivalência em relação aos Homens. A avaliação das propriedades psicométricas de cada Inventário revelou índices de fiabilidade interna aceitáveis: entre 0,83 e 0,87 para o AMI, e entre 0,37 e 0,74 para o ASI. A validade convergente foi também demonstrada, tendo sido reportadas correlações elevadas em particular entre as formas hostis de sexismo e outras medidas tradicionais de sexismo (Glick \& Fiske, 1996, 1999). A validade discriminante foi demonstrada pelos autores, nomeadamente através da associação entre sexismo moderno e racismo moderno (Glick \& Fiske, 1999), e também reportada em outros estudos (Fernandéz, Castro, \& Torrejón, 2001). De fato, a sua estrutura multidimensional foi replicada tanto em adaptações para a população Espanhola (Lameiras, Rodríguez, \& Sotelo, 2001) como para a população Brasileira (Formiga, Gouveia, \& Santos, 2002).

Em um estudo transcultural envolvendo 19 países e mais de 15.000 participantes (Glick et al., 2000), foi observado que as duas formas que caracterizam o Sexismo Ambivalente são de facto complementares e constantes em diferentes culturas. De uma forma geral, as flutuações nos níveis de hostilidade e de benevolência em relação às 
mulheres estão correlacionadas com os índices nacionais de desigualdade de género e de convencionalismo doméstico. Assim, em países como o Reino Unido, Austrália ou a Holanda, caracterizados por uma maior igualdade de oportunidades entre homens e mulheres, foram observados níveis de sexismo ambivalente mais baixos, em particular quando comparados com países como Cuba, África do Sul ou Nigéria, países onde as desigualdades sociais, que não só as de género, são mais prevalentes (Glick et al., 2000). De uma forma complementar, em um outro estudo envolvendo 16 países e 8.360 participantes (Glick et al., 2004) foi reportado que as atitudes ambivalentes em relação aos homens, em particular a crença que os homens deverão ter um papel dominante determinavam igualmente os níveis de desigualdade de género nos diferentes países.

Nestes estudos foi observado um índice de sexismo hostil em Portugal mais elevado do que em Espanha ou no Brasil, por exemplo. No gráfico de 19 países, Portugal situou-se no $9^{\circ}$ lugar dos países com maior índice de sexismo, entre Cuba onde foi encontrado o maior índice de hostilidade contra as mulheres, e a Holanda, com o índice mais baixo entre os países incluídos no estudo (Glick et al., 2000). Em contraste, os portugueses revelaram um baixo índice de hostilidade e de benevolência em relação aos homens, e comparativamente, bastante inferiores aos revelados por espanhóis, em um estudo que não incluiu dados da população brasileira (Glick et al., 2004). Estas atitudes sexistas, como tem sido reportado, estão fortemente associadas a valores sociais convencionais, religiosidade, tendência política, e a outras desigualdades sociais, como por exemplo racismo, classicismo, ou atitudes negativas em relação a homens gays e lésbicas (Belo, Gouveia, da Silva Raymundo, \& Marques, 2005; Christopher \& Mull, 2006; Fernandéz \& Castro, 2003; Formiga, 2007; Formiga, Araújo, \& Cavalcante, 2007; Formiga et al., 2002; Masser \& Abrams, 1999).

Tendo em conta o valor destes inventários na avaliação do preconceito sexista, e a falta de estudos e de instrumentos de medida na língua Portuguesa, o propósito deste estudo foi o de adaptar o Inventário de Sexismo Ambivalente e o Inventário de Ambivalência em relação aos Homens para Portugal, assim como de avaliar as características psicométricas e validade destes inventários neste contexto.

\section{Método}

\section{Participantes}

Um total de 258 indivíduos de ambos os sexos (80 homens e 178 mulheres), com uma idade média de 27 anos $(D P=10)$ foram recrutados em universidades Portuguesas. Do total da amostra, 59\% frequentavam a licenciatura, enquanto $41 \%$ frequentavam estudos pós-graduados (mestrado ou douramento). No que diz respeito aos dados sociodemográficos, a quase totalidade dos participantes identificou-se como heterossexual (94\%), 72\% como Católico, e 19\% como ateu. Em termos de tendência política, $26 \%$ dos participantes reportaram ser de um partido político de direita, $20 \%$ de um partido do centro, $13 \%$ de um partido de esquerda, e 39\% não identificaram nenhuma tendência partidária. Em termos de distribuição geográfica, todos os distritos Portugueses foram representados, embora a maioria dos participantes ser proveniente de Castelo Branco (centro do país; 23\%), Lisboa (18\%), e Porto (norte do país; $15 \%$ ).

\section{Instrumentos}

Os participantes responderam a um pequeno questionário sociodemográfico construído para este estudo, ao Inventário de Sexismo Ambivalente e ao Inventário de Ambivalência em relação aos Homens. O Inventário de Sexismo Ambivalente (ASI; Glick \& Fiske, 1996) é composto por 22 afirmações que medem as atitudes sexistas em relação às mulheres. Destas, 11 afirmações referem-se a uma forma benevolente de sexismo (por exemplo, "As mulheres deviam ser estimadas e protegidas pelos homens") e 11 afirmações referem-se a uma forma hostil de sexismo (por exemplo, "As mulheres procuram obter poder através de controlo sobre os homens").

O Inventário de Ambivalência em relação aos Homens (AMI; Glick \& Fiske, 1999) é composto por 20 itens que medem construtos semelhantes de sexismo (benevolente e hostil) dirigidos aos homens. Destes, 10 afirmações referem-se a uma forma benevolente de sexismo (por exemplo, "Os homens estão mais dispostos a correr riscos do que as mulheres") e 10 afirmações referem-se a uma forma hostil de sexismo (por exemplo, "Os homens irão sempre lutar para ter um maior controlo na sociedade do que as mulheres").

Para a adaptação dos inventários para a língua Portuguesa foram seguidas diretrizes internacionais para a adaptação transcultural de instrumentos de medida de fenómenos psicológicos (Beaton, Bombardier, Guillemin, \& Ferraz, 2000; Guillemin, Bombardier, \& Beaton, 1993). Um investigador bilingue, familiarizado com os conceitos, e dois investigadores bilingues não familiarizados com os conceitos, efetuaram três traduções da língua Inglesa para a língua Portuguesa; em seguida, as três traduções foram comparadas de forma a conseguir a uma tradução final parcimoniosa. A tradução final foi então enviada para um tradutor bilingue para efetuar uma retroversão da língua Portuguesa para a língua Inglesa. Finalmente, a tradução final em Português, a retroversão em Inglês, e o inventário original em Inglês foram comparados pelo autor principal do estudo, e foram feitas pequenas alterações a expressões linguísticas para facilitar a sua compreensão em Português. A tradução final foi posteriormente submetida a avaliação da sua validade facial em um focus group constituído por seis investigadores, familiarizados e não familiarizados com os conceitos.

Apesar de nas versões originais dos inventários as afirmações serem medidas numa escala de Likert de 6 pontos, para este estudo foi utilizada uma escala de Likert de 5 pontos (de 1 = "discordo totalmente" a 5 = "concordo totalmente"), por forma a permitir que os participantes 
Costa, P. A., Oliveira, R., Pereira, H. \& Leal, I. (2015). Adaptação dos Inventários de Sexismo Moderno para Portugal: O Inventário de Sexismo Ambivalente e o Inventário de Ambivalência em Relação aos Homens.

escolhessem um ponto intermédio e assim averiguar a sensibilidade individual dos itens.

\section{Procedimentos}

Os participantes foram recrutados online, em diversas universidades Portuguesas, com o único critério de inclusão frequentarem a universidade. Os participantes foram contactos por correio eletrónico distribuído pela lista de alunos das universidades, e convidados a participar num estudo sobre "Crenças sobre as relações entre homens e mulheres". Na mensagem foi apresentada uma breve descrição do estudo e seus objetivos, assim como uma ligação que direcionava os participantes para o questionário online alojado em Google Docs. Na primeira página do questionário foi novamente dada a informação pertinente sobre o estudo, e solicitado o consentimento informado dos participantes. Este estudo foi aprovado em Comité de Ética da Unidade de Investigação em Psicologia e Saúde (ISPA - Instituto Universitário), e respeitou os princípios deontológicos definidos pela Ordem dos Psicólogos Portugueses (OPP), assim como as diretrizes definidas pela Associação de Psicologia Americana (APA) para a condução de estudos com seres humanos.

\section{Análise dos Dados}

Os questionários foram analisados com o programa IBM SPSS, versão 20, e com o programa IBM SPSS Amos, versão 20. A estatística descritiva e as análises correlacionais foram realizadas com o IBM SPSS, e as análises fatoriais confirmatórias foram realizadas por recurso ao IBM SPSS Amos.

\section{Resultados}

Antes de proceder às análises fatoriais confirmatórias e de avaliar o ajustamento dos modelos de equações estruturais foi avaliada a normalidade dos dados. Para o Inventário de Sexismo Ambivalente, os valores de Kurtose $(|\mathrm{Ku}|)$ variaram entre $-0,028$ e $-1,375$ e os valores de Assimetria ( $|\mathrm{Sk}|)$ entre 0,088 e 0,989; para o Inventário de Ambivalência em relação aos Homens, os valores de $|\mathrm{Ku}|$ variaram entre $-1,242$ e $-0,122$ e os valores de $|\mathrm{Sk}|$ entre -0,336 e 1,375, indicando uma distribuição normal dos dados (Kline, 2010). A estatística de distância de Mahalanobis $\left(\mathrm{D}^{2}\right)$ foi também analisada para detetar a presença de outliers multivariados, mas nenhum outlier multivariado foi encontrado em nenhum dos inventários.

\section{Análise Fatorial Confirmatória do Inventário de Sexismo Ambivalente}

O modelo de $2^{\mathrm{a}}$ ordem proposto pelos autores do inventário foi testado através da análise fatorial confirmatória. A análise preliminar revelou que dois tens não pesaram significativamente no seu fator Sexismo Hostil (item 18: "Existem, na realidade, muito poucas mulheres que se divertem a provocar homens mostrando-se sexualmente disponíveis, e depois recusando os avanços masculinos" e item 21: "As feministas estão a fazer exigências inteiramente razoáveis aos homens").

Após a eliminação destes dois itens, a estatística do Qui Quadrado foi ainda assim significativa $\left(\chi^{2}=299,193, g l=\right.$ $165 ; p<0,001)$, embora diversos autores tenham mostrado que a estatística do Qui Quadrado não é um índice adequado em muitos casos, em particular quando as amostras são de grande dimensão (Bentler, 2007; Hayduk, 1996). Outros índices de ajustamento revelaram um modelo com um bom ajustamento aos dados ( $\mathrm{CFI}=0,92$; GFI $=0,90$; RMSEA $=0,05)$. Os restantes itens pesaram significativamente no seu fator, e a validade fatorial individual foi observada em todos os casos, com exceção dos itens 3 e 7 para os quais os pesos fatoriais estandardizados foram inferiores a 0,5 (Tabela 1).

Tabela 1

Fatores, Itens, e Pesos Fatoriais do ASI

\begin{tabular}{llr}
\hline $\mathrm{N}^{\mathrm{o}}$ Item & Fator / Item & Peso Fatorial
\end{tabular}

\section{Sexismo Hostil}

2 Muitas mulheres estão, na realidade, à procura de favores especiais, tais como políticas de contratação que as favoreçam em detrimento dos homens, sob o pretexto de requererem "igualdade"

4 A maioria das mulheres interpreta comentários ou atos inocentes como sendo sexistas 
11 As mulheres procuram obter poder através de controlo sobre os homens $\quad 0,75$

14 As mulheres exageram os problemas que têm no trabalho $\quad 0,64$

15 Quando uma mulher consegue que um homem se comprometa com ela, geralmente tenta $\quad 0,74$ dar-lhe rédea curta

16 Quando as mulheres perdem com homens numa competição justa, elas costumam $\quad 0,70$ queixar-se de terem sido discriminadas

18 Existem, na realidade, muito poucas mulheres que se divertem a provocar homens mostrando-se sexualmente disponíveis, e depois recusando os avanços masculinos

21 As feministas estão a fazer exigências inteiramente razoáveis aos homens

\section{Sexismo Benevolente}

Paternalismo Protetor

3 Num desastre, as mulheres não deviam, necessariamente, ser resgatadas antes dos homens $\quad 0,30$

9 As mulheres deviam ser estimadas e protegidas pelos homens 0,55

17 Uma boa mulher deve ser colocada num pedestal pelo seu homem $\quad 0,64$

20 Os homens devem estar dispostos a sacrificar o seu próprio bem-estar para garantirem $\quad 0,55$ a segurança financeira para as mulheres nas suas vidas

Diferenciação de Género Complementar

$8 \quad$ Muitas mulheres têm uma qualidade de pureza que poucos homens possuem 0,68

19 As mulheres, comparadas com os homens, tendem a ter uma sensibilidade moral superior $\quad 0,73$

22 As mulheres, comparadas com os homens, tendem a ter um sentido mais refinado de cultura $\quad 0,68$ e bom gosto

\section{Intimidade Heterossexual}

1 Não importa o quão realizado seja, um homem não é verdadeiramente completo enquanto $\quad 0,81$ pessoa se não tiver o amor de uma mulher

6 As pessoas são muitas vezes verdadeiramente felizes na vida sem estarem romanticamente $\quad 0,55$ envolvidas com uma pessoa do sexo oposto

12 Todo o homem devia ter uma mulher que ele adore $\quad 0,71$

13 Os homens são completos sem mulheres $\quad 0,66$

\section{Análise Fatorial Confirmatória do Inventário}

de Ambivalência em relação aos Homens

O mesmo procedimento foi adotado para a análise fatorial confirmatória do Inventário de Ambivalência em relação aos Homens. Todos os itens pesaram significativamente no seu fator, e todos os fatores de $1^{\mathrm{a}}$ ordem pesaram significativamente no respetivo fator de $2^{\mathrm{a}}$ ordem. A estatística do Qui Quadrado foi significativa $\left(\chi^{2}=314,974, g l=\right.$ $162, p<0,001)$, embora os restantes índices de ajustamento tenham revelado um bom ajustamento do modelo $(\mathrm{CFI}=$ 0,91 ; GFI $=0,89$; RMSEA $=0,06$ ). A validade fatorial individual foi observada em todos os casos (Tabela 2). 
Costa, P. A., Oliveira, R., Pereira, H. \& Leal, I. (2015). Adaptação dos Inventários de Sexismo Moderno para Portugal: O Inventário de Sexismo Ambivalente e o Inventário de Ambivalência em Relação aos Homens.

Tabela 2

Fatores, Itens, e Pesos Fatoriais do AMI

\begin{tabular}{ccc}
\hline $\mathrm{N}^{\mathrm{o}}$ & Fator / Item & Peso \\
Item & & Fatorial \\
\hline
\end{tabular}

Hostilidade em relação aos Homens

\section{Ressentimento do Paternalismo}

9 Os homens irão sempre lutar para ter um maior controlo na sociedade do que as mulheres

Mesmo os homens que alegam ser sensíveis aos direitos das mulheres, na realidade, querem ter um relacionamento tradicional em casa, com a mulher a desempenhar

11 a maioria das tarefas domésticas e a cuidar das crianças

A maioria dos homens dizem-se apoiantes da igualdade das mulheres, mas não conseguem lidar com

15 uma mulher como igual

Diferenciação de Género Compensatória

6 Os homens estariam perdidos neste mundo se as mulheres não estivessem lá para os guiar

8 Os homens agem como bebés quando estão doentes

17 Na realidade, a maioria dos homens são como crianças

Hostilidade Heterossexual

Um homem que está sexualmente atraído por uma mulher normalmente não tem

2 problemas morais em fazer o que for necessário para a levar para a cama

Quando os homens agem de forma a "ajudar" as mulheres estão, frequentemente, a tentar provar que

4 são melhores do que elas

14 Os homens geralmente tentam dominar as conversas quando falam com mulheres

A maioria dos homens assedia sexualmente mulheres, mesmo que de formas subtis, quando estão numa

19 posição de poder sobre elas

Benevolência em relação aos Homens

Maternalismo

Mesmo quando ambos os membros de um casal trabalham, a mulher deve ser mais atenta

1 a cuidar do seu homem em casa

10 Os homens são essencialmente úteis para garantirem a segurança financeira das mulheres

As mulheres deviam cuidar do seu homem em casa, porque os homens se deixariam ir abaixo se

20 tivessem de tomar conta de si próprios

Diferenciação de Género Complementar

Os homens são menos propensos a perder o controlo em situações de emergência

3 do que as mulheres

13 Os homens estão mais dispostos a colocarem-se em perigo para proteger outros

18 Os homens estão mais dispostos a correr riscos do que as mulheres

Intimidade Heterossexual

5 Toda a mulher precisa de um parceiro do sexo masculino que a estime

Uma mulher nunca se sentirá verdadeiramente realizada se não tiver uma relação, comprometida e de

7 longa duração, com um homem

12 Toda a mulher devia ter um homem que adore

16 As mulheres são incompletas sem os homens 


\section{Fiabilidade Compósita e Validade Discriminante}

A consistência e a fiabilidade internas foram adequadas para todos os fatores de ambos os inventários, ASI e AMI, assim como apresentaram em todos os casos evidência de validade discriminante (Tabela 3 ).

\section{Validade Convergente e Correlações com Variáveis} Demográficas

De acordo com as diretrizes propostas pelos autores dos inventários ASI e AMI, foram avaliadas as correlações simples bivariadas e as correlações parciais entre os inventários e os seus subfactores, e entre estes e as variáveis sociodemográficas comummente preditoras de atitudes sexistas: sexo, idade, anos de escolaridade, religião, e tendência política. Idade e escolaridade foram medidas como variáveis contínuas. Género, religião e política foram codificadas como variáveis dummy; sexo: $0=$ mulher, $1=$ homem; religião: $0=$ não católico, $1=$ católico; inclinação política: $0=$ esquerda; $1=$ direita (Tabela 5 ).

Nas correlações parciais, por forma a obter correlações mais puras e não confundidas por outra forma de sexismo, cada subfactor (sexismo hostil ou sexismo benevolente) foi correlacionado controlando a correlação do outro subfactor. As correlações bivariadas revelaram que os inventários estavam significativamente e fortemente associados $(r=$ 0,718) (Tabela 4).

Tabela 3

Fiabilidade Compósita (FC), Validade Discriminante (Variância Extraída da Média; AVE), Média (M), e DesvioPadrão (DP) dos Inventários ASI e AMI

\begin{tabular}{lcccc}
\hline & FC & VEM & $M$ & $D P$ \\
\hline ASI & 0,93 & 0,41 & 2,79 & 0,60 \\
Sexismo Hostil (SH) & 0,86 & 0,41 & 2,70 & 0,77 \\
Sexismo Benevolente (SB) & 0,88 & 0,41 & 2,75 & 0,68 \\
AMI & 0,94 & 0,44 & 2,60 & 0,61 \\
Hostilidade em relação Homens (HH) & 0,86 & 0,38 & 2,74 & 0,71 \\
Benevolência em relação Homens (BH) & 0,91 & 0,50 & 2,45 & 0,74 \\
\hline
\end{tabular}

Tabela 4

Correlações entre o ASI, AMI, e Respetivos Subfatores

\begin{tabular}{lcccccc}
\hline & \multicolumn{5}{c}{ Correlações simples bivariadas } \\
\cline { 2 - 7 } & 1 & 2 & 3 & 4 & 5 & 6 \\
\hline 1. ASI & - & & & & \\
$2 . \mathrm{SH}$ & $0,82^{*}$ & & & & \\
3. SB & $0,85^{* *}$ & $0,41^{*}$ & & & \\
4. AMI & $0,72^{*}$ & $0,50^{*}$ & $0,69 *$ & - & \\
$5 . \mathrm{HH}$ & $0,42^{*}$ & $0,27^{*}$ & $0,42^{*}$ & $0,84^{*}$ & - & \\
$6 . \mathrm{BH}$ & $0,79^{*}$ & $0,57^{*}$ & $0,75^{*}$ & $0,85^{*}$ & $0,43^{*}$ & - \\
\hline
\end{tabular}

${ }^{*} p<0,005 ; * * p<0,001$.

Sexo estava significativamente e positivamente correlacionado com o inventário ASI $(r=0,303)$, com sexismo hostil ( $r=0,296$, controlando sexismo benevolente) e com benevolência em relação aos homens $(r=0,373$, controlando hostilidade em relação aos homens). Em contraste, sexo correlacionou significativamente mas de forma negativa com hostilidade em relação aos homens $(r=-0,327$, controlando benevolência em relação aos homens).

Idade correlacionou positivamente com hostilidade em relação aos homens ( $r=0,187$, controlando benevolência) e negativamente com benevolência em relação aos homens $(r=-0,195)$. Educação correlacionou negativamente com 
Costa, P. A., Oliveira, R., Pereira, H. \& Leal, I. (2015). Adaptação dos Inventários de Sexismo Moderno para Portugal: O Inventário de Sexismo Ambivalente e o Inventário de Ambivalência em Relação aos Homens.

sexismo hostil ( $r=0,201$; controlando sexismo benevolente), sexismo benevolente $(r=-0,281$, controlando hostilidade), e com o AMI global $(r=-0,133)$.

Religião correlacionou positivamente com o ASI $(r=$ $0,197)$ e com o AMI $(r=0,156)$ globais. Correlacionou igualmente de forma positiva com sexismo benevolente $(\mathrm{r}$ $=0,186$, controlando hostilidade) e com benevolência em relação aos homens ( $r=0,229$, controlando hostilidade). Afiliação política não se encontrou correlacionada significativamente com nenhum dos inventários ou subfactores.

Tabela 5

Correlações entre o ASI, AMI, Subfatores e Variáveis Sociodemográficas

\begin{tabular}{lccccc}
\hline & Sexo & Idade & Escolaridade & Religião & Afiliação Política \\
\hline 1. ASI & $0,30^{*}$ & $-0,04$ & $-0,08$ & $0,20^{* *}$ & 0,10 \\
2. SH & $0,32^{*}$ & 0,01 & $-0,01$ & $0,14^{* *}$ & 0,15 \\
(2a) SH (controlando SB) & $0,30^{*}$ & $-0,03$ & $0,20^{* *}$ & 0,08 & 0,15 \\
3. SB & $0,20^{*}$ & $-0,07$ & $-0,12^{* *}$ & $0,20^{*}$ & 0,03 \\
(3a) BS (controlando SH) & 0,09 & $-0,01$ & $-0,28^{*}$ & $0,19^{* *}$ & $-0,04$ \\
4. AMI & 0,04 & $-0,08$ & $-0,13^{* *}$ & $0,16^{* *}$ & 0,07 \\
5. HH & $-0,16^{* *}$ & $-0,01$ & $-0,12$ & 0,11 & $-0,01$ \\
(5a) HH (controlando BH) & $-0,33^{*}$ & $0,19^{* *}$ & $-0,05$ & 0,04 & $-0,07$ \\
6. BH & $0,22^{*}$ & $-0,14^{* *}$ & $-0,11$ & $0,15^{* *}$ & 0,12 \\
(6a) BM controlando HH) & $0,37^{*}$ & $-0,20 * *$ & $-0,12$ & $0,23^{* *}$ & 0,14
\end{tabular}

${ }^{*} p<0,005 ; * *<0,001$.

\section{Discussão}

Os objetivos deste estudo foram a adaptação do Inventário de Sexismo Ambivalente (ASI) e do Inventário de Ambivalência em relação aos Homens (AMI), e a avaliação das suas qualidades psicométricas em Portugal. Os resultados suportaram a multidimensionalidade do construto de Sexismo Ambivalente, baseada na diferenciação entre Hostilidade e Benevolência, tanto em relação às mulheres como aos homens. Foi também observado que o modelo de sexismo multidimensional proposto por Glick e Fiske $(1996,1999)$ é confiável e constante em diferentes culturas. A replicação deste modelo em Portugal revelou a que os inventários mantêm a estrutura fatorial proposta pelos autores à semelhança de outros estudos de validação (Formiga et al., 2002; Lameiras et al., 2001).

A análise fatorial confirmatória do Inventário de Sexismo Ambivalente revelou um bom ajustamento do modelo, tendo sido obtidos índices de ajustamento muito semelhantes aos reportados pelos autores deste inventários, e índices de consistência interna superiores (Glick \& Fiske, 1996). Contudo, dois itens do fator sexismo hostil foram eliminados, provavelmente devido a problemas de adaptação cultural. No primeiro caso, o problema deverá estar na tradução do item para Português, uma vez que a afirmação foi apresentada de uma forma complexa, e por isso pouco clara: "Existem, na realidade, muito poucas mulheres que se divertem a provocar homens mostrando-se sexualmente disponíveis, e depois recusando os avanços masculinos". Nos procedimentos de tradução e adaptação dos inventários os tentou-se manter o mais possível fiel à formulação original das afirmações, pelo que algumas expressões idiomáticas poderão não ser facilmente compreendidas. Sugerimos que este item poderia ser melhorado invertendo a intenção e simplificando a frase, como por exemplo, "Existem muitas mulheres que se divertem a provocar homens mostrando-se sexualmente disponíveis, e depois recusam os avanços masculinos". Esta alteração resultaria, contudo, na inversão da cotação do item.

No segundo caso, a explicação poderá estar relacionada com o entendimento dos participantes do conceito de $\mathrm{Fe}$ minismo, podendo as ideias preconcebidas associadas ao movimento feminista afetar a forma como os participantes compreendem a afirmação "As feministas estão a fazer exigências inteiramente razoáveis aos homens". É ainda comum no imaginário social entender o feminismo, e a mulher feminista, através de ideias preconceituosas que associam este movimento a uma tentativa de controlo sobre os homens, e/ou a mulheres masculinizadas ou lésbicas (Saldanha, Scarparo, \& Strey, 2013). Se "feministas" for entendido com este tipo de preconceito, será de esperar que a avaliação dos participantes decorra no sentido de estas exigências não serem justas. Em estudos futuros deverão ser testadas novas formulações destas duas afirmações por forma a corrigir os problemas encontrados. Não obstante, foi demonstrada a validade convergente e discriminante de cada fator. 
A análise fatorial confirmatória do Inventário de Ambivalência em relação aos Homens revelou igualmente um bom ajustamento do modelo aos dados, assim como índices de ajustamento semelhantes aos dos reportados no estudo original (Glick \& Fiske, 1999). Em comparação com o ASI, o AMI revelou melhores qualidades psicométricas no que respeita à validade e fiabilidade interna dos itens e subfactores. Os inventários ASI e AMI revelaram estar fortemente correlacionados, fornecendo evidência de validade do construto uma vez que ambos os inventários medem construtos semelhantes ainda que dirigidos a diferentes sexos. Os subfactores de cada inventário revelaram também fortes correlações entre si, à exceção do subfactor Hostilidade em relação aos homens quando controlado o subfactor Ambivalência em relação aos homens. Assim, ao controlar o subfactor Benevolência, obtêm-se uma medida mais pura de sexismo hostil. De facto, a correlação obtida entre o ASI e o AMI deveu-se largamente ao subfactor Benevolência. Esta correlação foi mais elevada no presente estudo, quando comparado a um estudo semelhante com estudantes universitários Espanhóis (Fernández, Castro, \& Lorenzo, 2004), sugerindo uma atitude geral mais genderizada e rígida em Portugal do que em Espanha.

Evidência adicional de validade de construto foi revelada pelas correlações moderadas encontradas entre os inventários e as diferentes variáveis sociodemográficas. No que diz respeito ao sexo, os homens revelaram níveis mais elevados de sexismo do que as mulheres, assim como níveis mais elevados de benevolência em relação aos homens. De salientar, ser católico encontrou-se associado a uma forma benevolente de sexismo, tanto dirigido aos homens como às mulheres, mas não com hostilidade, $o$ que está de acordo com estudos anteriores da associação entre religiosidade e sexismo (Burn \& Busso, 2005; Glick, Lameiras, \& Castro, 2002). Afiliação política não correlacionou significativamente com nenhuma forma de sexismo, apesar de a literatura indicar que uma ideologia conservadora, nomeadamente o autoritarismo de direita, é um importante preditor de Sexismo Ambivalente (Christopher \& Mull, 2006).

Também de acordo com a literatura (Glick \& Fiske, 1996; Sidanius, Pratto, \& Bobo, 1996), os participantes com maior escolaridade revelaram maior hostilidade em relação às mulheres, enquanto os participantes com menor escolaridade revelaram mais benevolência em relação aos homens e às mulheres. Contudo, (Glick \& Fiske, 1996) e contrário à literatura, os participantes mais jovens revelaram atitudes mais benevolentes em relação aos homens, e os participantes mais velhos atitudes mais hostis em relação aos homens. Tem sido reportado que pessoas mais jovens tendem a adotar atitudes mais polarizadas, hostis ou igualitárias (Glick \& Fiske, 1996), e atitudes mais benevolentes são mais expressivas em pessoas já em idade adulta. Neste estudo, apesar de os participantes serem estudantes universitárias, a média de idades foi de 27 anos, e cerca de $40 \%$ destes estarem a níveis pós-graduados, o que poderá explicar este resultado aparentemente contraditório.
Em suma, os resultados do presente estudo suportam a estrutura multidimensional e confiabilidade do Inventário de Sexismo Ambivalente e do Inventário de Ambivalência em relação aos Homens em Portugal. Os resultados revelaram que as atitudes sexistas se encontram associadas a convencionalismo social, medido através de religiosidade e tendência política. Foram também encontrados efeitos de sexo e de idade, uma vez que tanto os homens como as mulheres revelaram maior hostilidade em relação ao sexo oposto, e que a benevolência em relação aos homens aumentava com a idade enquanto a hostilidade em relação aos homens diminuía. Tanto o ASI como o AMI mostraram-se medidas fiáveis e confiáveis na avaliação dos complexo construto de Sexismo Moderno, ou Ambivalente. Mais pesquisas são necessárias para explorar as relações genderizadas entre homens e mulheres e as suas implicações.

Apesar dos resultados obtidos no presente estudo evidenciarem as qualidades psicométricas dos inventários de sexismo moderno em Portugal, a amostra deste estudo foi retirada de uma população universitária e por isso com mais formação do que a população geral, representando um importante limitação do estudo. Futuros estudos deverão avaliar as qualidades destes inventários com a população geral, por forma a reunir evidências adicionais da validade e precisão dos inventários. Mais, o fato de o presente estudo ter sido conduzido online representa uma outra limitação, uma vez que desta forma não é possível controlar as circunstâncias em que os participantes preencheram os questionários, ou a sua compreensão das afirmações.

\section{Conclusão}

O presente estudo apresenta algumas importantes contribuições para o conhecimento científico do sexismo moderno. Apesar de existirem anteriores estudos em que os inventários foram utilizados (Glick et al., 2000; Glick et al., 2004), não existe nenhum artigo disponível que apresente o processo de adaptação e desenvolvimento do ASI e do AMI que demonstrem a sua validade de conteúdo e de construto, assim como as suas características psicométricas que permitem concluir que as versões dos Inventários em língua Portuguesa mantêm uma estrutura fatorial e características psicométricas muito semelhantes às originais. Mais, as versões em língua Portuguesa sugerem que estas apreendem o construto de sexismo moderno pretendido. Contudo, o facto de dois dos itens do Inventário de Sexismo Ambivalente não terem tido um peso significativo, revela que estes deverão ser reavaliados em estudos futuros. Assim, propõe-se com este estudo versões fiáveis dos Inventários de Sexismo Moderno em Portugal.

Os estudos de adaptação e validação de instrumentos de medida de preconceito, neste caso particular de preconceito sexista, são essenciais para a avaliação das crenças e valores das pessoas que permitam desenvolver medidas concretas que combatam as discriminações existentes entre homens e mulheres e que promovam uma maior igualdade de oportunidades entre os géneros. 
Costa, P. A., Oliveira, R., Pereira, H. \& Leal, I. (2015). Adaptação dos Inventários de Sexismo Moderno para Portugal: O Inventário de Sexismo Ambivalente e o Inventário de Ambivalência em Relação aos Homens.

\section{Referências}

Allport, G. W. (1954). The nature of prejudice. Reading, UK: Addison-Wesley.

Beaton, D. E., Bombardier, C., Guillemin, F., \& Ferraz, M B. (2000). Guidelines for the process of cross-cultural adaptation of self-report measures. Spine, 25, 3186-3191. doi:10.1097/00007632-200012150-00014

Belo, R. P., Gouveia, V. V., da Silva Raymundo, J., \& Marques, C. M. C. (2005). Correlatos valorativos do sexismo ambivalente. Psicologia: Reflexão e Crítica, 18, 7-15. doi:10.1590/ S0102-79722005000100003

Bentler, P. M. (2007). On tests and indices for evaluationg structural models. Personality and Invidual Differences, 42, 825-829. doi:10.1016/j.paid.2006.09.024

Burn, S. M., \& Busso, J. (2005). Ambivalent sexism, scriptural literalism, and religiosity. Psychology of Women Quarterly, 29, 412-418. doi:10.1111/j.1471-6402.2005.00241.x

Christopher, A. N., \& Mull, M. S. (2006). Conservative ideology and ambivalent sexism. Psychology of Women Quarterly, 30, 223-230. doi:10.1111/j.1471-6402.2006.00284.x

Fernandéz, M. L., \& Castro, Y. R. (2003). Assessment of ambivalent sexism in Galician students. Acción Psicológica, 2 131-136. doi:10.5944/ap.2.2.526

Fernández, M. L., Castro, Y. R., \& Lorenzo, M. G. (2004). Evolution of hostile sexism and benevolent sexism in a Spanish sample. Social Indicators Research, 66, 197-211. doi:10.1023/B:SOCI.0000003553.30419.f1

Fernandéz, M. L., Castro, Y. R., \& Torrejón, M. J. S. (2001). Sexism and racism in a Spanish sample of secondary school students. Social Indicators Research, 54, 309-328. doi:10.1023/A:1010871706454

Formiga, N. S. (2007). Valores humanos e sexismo ambivalente. Revista do Departamento de Psicologia - UFF, 19, 381-396. doi:10.1590/S0104-80232007000200009

Formiga, N. S., Araújo, T. T. V., \& Cavalcante, C. P. S. (2007). A manutenção da discriminação feminina no contexto brasileiro: Um estudo sobre a fidedignidade do sexismo ambivalente. Estudos e Pesquisas em Psicologia, 7, 56-67.

Formiga, N. S., Gouveia, V. V., \& Santos, M. N. (2002). Inventário de sexismo ambivalente: Sua adaptação e relação com o gênero. Psicologia em Estudo, 7, 105-111. doi:10.1590/ S1413-73722002000100013

Glick, P., \& Fiske, S. T. (1996). The Ambivalent Sexism Inventory: Differentiating hostile and benevolent sexism. Journal of Personality and Social Psychology, 70, 491-512. doi:199603014-006

Glick, P., \& Fiske, S. T. (1997). Hostile and benevolent sexism: Measuring ambivalent sexist attitudes toward women. Psychology of Women Quarterly, 21, 119-135. doi:10.1111/j.1471-6402.1997.tb00104.x

Glick, P., \& Fiske, S. T. (1999). The Ambivalence toward Men Inventory: Differentiating hostile and benevolent beliefs about men. Psychology of Women Quarterly, 23, 519-536. doi:10.1111/j.1471-6402.1999.tb00379.x

Glick, P., \& Fiske, S. T. (2001). An ambivalent alliance: Hostile and benevolent sexism as complementary justifications for gender inequality. American Psychologist, 56, 109-118. doi:2001-00159-001

Glick, P., Fiske, S. T., Mladinic, A., Saiz, J. L., Abrams, D., Masser, B., ...López, W. L. (2000). Beyond prejudice as simple antipathy: Hostile and benevolent sexism across cultures. Journal of Personality and Social Psychology, 79, 763-775. doi:10.1037/0022-3514.79.5.763
Glick, P., Fiske, S. T., Masser, B., Manganelli, A. M., Huang, L., Castro, Y. R., ...Wells, R. (2004). Bad but bold: Ambivalent attitudes toward men predict gender inequality in 16 nations. Journal of Personality and Social Psychology, 86, 713-728. doi:2004-13298-005

Glick, P., Lameiras, M., \& Castro, Y. (2002). Education and catholic religiosity as predictors of hostile and benevolent sexism toward women and men. Sex Roles, 47, 433-441. doi:10.1023/A:1021696209949

Guillemin, F., Bombardier, C., \& Beaton, D. (1993). Crosscultural adaptation of health-related quality of life measures: Literature review and proposed outlines. Journal of Clinical Epidemiology, 46, 1417-1432. doi:10.1016/08954356(93)90142-N

Hayduk, L. A. (1996). LISREL: Issues, debates and strategies Batimore, MD: Hopkins University Press.

Kline, R. B. (2010). Principles and practice of structural equation modeling ( $3^{\text {nd }}$ ed.). New York: Guilford Press.

Lameiras, M., Rodríguez, Y., \& Sotelo, M. J. (2001). Sexism and racism in a Spanish sample of secondary school students. Social Indicators Research, 54(3), 309-328.

Masser, B., \& Abrams, D. (1999). Contemporary sexism: The relationships among hostility, benevolence, and neosexism. Psychology of Women Quarterly, 23, 503-517. doi:10.1111/j.1471-6402.1999.tb00378.x

Saldanha, M., Scarparo, H. B. K., \& Strey, M. N. (2013). Porque não somos todas feministas? Diálogo (Canoas), 22, 107-116.

Sidanius, J., Pratto, F., \& Bobo, L. (1996). Racism, conservatism, affirmative action, and intellectual sophistication: A matter of principled conservatism or group dominance? Journal of Personality and Social Psychology, 70, 476-490. doi:199603014-005

Tajfel, H. (1981). Social identity and intergroup relations. London: Combridge University Press. 